\title{
Acoustic Emission Monitoring of Laser Shock Peening by Detection of Underwater Acoustic Wave
}

\author{
Tomoki Takata $^{1,{ }^{*} 1,{ }^{* 2}}$, Manabu Enoki ${ }^{1}$, Pornthep Chivavibul ${ }^{1}$, Akinori Matsui ${ }^{2}$ and Yuji Kobayashi ${ }^{2}$ \\ ${ }^{1}$ Department of Materials Engineering, The University of Tokyo, Tokyo 113-8656, Japan \\ ${ }^{2}$ SHINTOKOGIO, LTD., Nagoya 460-0003, Japan
}

\begin{abstract}
Acoustic Emission (AE) technique is one of the nondestructive methods to evaluate the size, location and generation time of deformation or damage of material in real times. Generally AE sensors are directly attached on the surface of the component to detect AE wave, however this method brings about inconvenient setting to many industrial processes. In the present study, an arrangement of AE sensors was investigated to monitor laser shock peening (LSP). Instead of direct attachment of the sensors on the target, several AE sensors were located in the water layer to detect acoustic wave propagating through the water. The results showed that the sensor arrangement has a good performance to monitor LSP. Impact pressures during LSP process were obtained from detected AE waveforms by deconvolution technique. In addition, with AE measurement, sample surface was observed by high speed camera and investigated phenomena during LSP process.

[doi:10.2320/matertrans.M2015401]
\end{abstract}

(Received November 2, 2015; Accepted February 15, 2016; Published April 25, 2016)

Keywords: laser shock peening, acoustic emission method, inverse analysis, cavitation bubble, laser induced breakdown

\section{Introduction}

Laser shock peening (LSP) process is one of surface treatments to induce residual compressive stress into material surface to improve fatigue property of material. Laser is irradiated on metal surface under confinement layer such as water or glass to generate the high pressure near material surface by laser ablation in LSP process. LSP has several advantages, for example LSP process is available to give surface treatment at only desired area. However, the effects of laser irradiation on materials and the phenomena during LSP process near laser irradiation position are not well understood yet. The residual compressive stress in depth direction is generally used to evaluate the degree of LSP process. Measurement of the residual stress can be carried out by both destructive and non-destructive methods. It is inconvenience for manufacture that peened components have to be cut or electrochemically polished for the destructive methods. It is desired that the nondestructive methods provide capability for in-situ evaluation during LSP process.

There are several nondestructive methods to evaluate the degree of LSP such as velocity interferometer system for any reflector $(\mathrm{VISAR})^{1)}$ and X-ray stress measurement ${ }^{2}$. VISAR method applies continuous laser onto the opposite side of peened surface to measure the surface wave velocity, which is determined from an interference fringe between the initial and reflected laser based on Doppler effect. Obtained surface wave velocity coupling with Rankine-Hugoniot equation is used to calculate the pressure in the peened surface. However, this method can be applied to thin sample with the thickness less than about $1 \mathrm{~mm}$. X-ray stress measurement gives the residual stress value near the material surface. The polishing of surface is required to obtain the stress profile in the depth direction.

Acoustic emission (AE) is one of the nondestructive methods to evaluate the size, location and generation time of de-

\footnotetext{
${ }^{* 1}$ Corresponding author, E-mail: takata@ rme.mm.t.u-tokyo.ac.jp

${ }^{* 2}$ Graduate Student, The University of Tokyo
}

formation and damage in real-time by detecting elastic wave with the following phenomenon. AE sources, such as impact with foreign objects ${ }^{3)}$, fatigue cracks initiation and growth ${ }^{4)}$, rubbing $^{5)}$, and fiber breakage ${ }^{6)}$, can generate the propagation of elastic waves. Most of the information about damage and deformation of structures can be obtained by AE method. Due to its advantages in in-situ monitoring and source localization, AE method has been used in a variety of fields such as material ${ }^{7)}$ and manufacturing processes ${ }^{8)}$, especially in plate-like structures which are commonly used in civil and aerospace. LSP is the process with elastic wave by laser ablation. So, AE method has been successfully applied to LSP method to monitor the peening phenomena and the impact force $^{9)}$. In the previous study, the AE sensors were directly attached on the sample surface, therefore the wave propagation behavior was affected by the geometry and properties of the sample. In addition, attaching sensor on the sample is not available for process monitoring because of fixing one $\mathrm{AE}$ sensor on one material.

To overcome this problem with sensor attaching, the following approach was proposed in this study. Instead of the attaching sensor on sample surface, several AE sensors were set in the water at fix positions independent from sample to detect AE wave propagating though water. This approach is able to provide a flexibility of specimen setting and a capability of on-line control to process monitoring. The present study is to evaluate the performance of new AE arrangement for monitoring of LSP process. The results were compared with those obtained from direct attachment of AE sensor on the sample surface.

\section{Experimental Methods}

\subsection{Material}

A7075 aluminum alloy, which is used in aerospace field, was used as a sample for the present study. The samples were prepared by machining into dimensions of $35 \times 35 \times 20 \mathrm{~mm}$ and their surfaces were polished up to $3-\mu \mathrm{m}$ alumina slurry. The sample was then put into the water tank and fixed onto 


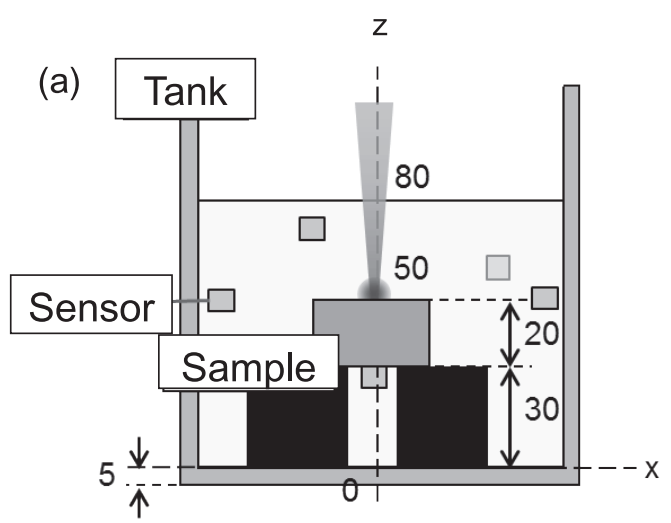

(b)

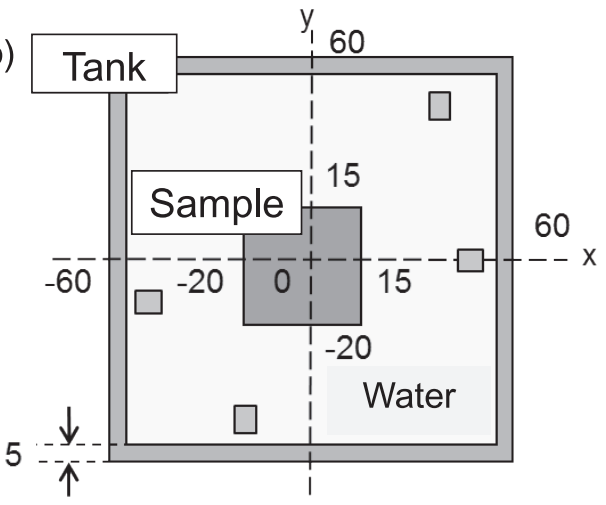

\section{Dimensions: $\mathrm{mm}$}

Fig. 1 Experimental setup 1 of laser shock peening (a) $x-z$ plane and (b) $x-y$ plane.

3D-controllable jig.

\subsection{Laser shock peening and $\mathrm{AE}$ measurement}

A Q-switched Nd:YAG laser with a wave length of $532 \mathrm{~nm}$, a pulse width of 3 to $5 \mathrm{~ns}$, and maximum energy of $100 \mathrm{~mJ}$, was used for LSP. This laser was focused onto the desired position of sample surface. At the opposite side of the specimen surface, an AE sensor (PICO, Physical Acoustics Corp.) was attached to detect $\mathrm{AE}$ waveforms propagating through the sample. Cables of AE sensors, which were soaked in the water, were covered by the waterproof paste. Continuous Wave Memory (CWM) system, which is developed by our research group ${ }^{10)}$, was applied to record and analysis waveforms. This system provides a capability to memorize all waveforms continuously at the sampling rate of $10 \mathrm{MHz}$.

Two kinds of experiment setup were performed. A schematic diagram of the first experiment setup is shown in Fig. 1. The side and top views are shown in Fig. 1(a) and (b), respectively. In order to detect AE waveforms propagating through the water, four $\mathrm{AE}$ sensors were located in the water layer at different $(\mathrm{x}, \mathrm{y}, \mathrm{z})$ positions as follows: $(-50,7.5,50),(35,50$, $60),(-15,-50,70)$ and $(50,0,50)$. In this experiment, the center bottom of the tank was defined as $(0,0,0)$ position. The laser was focused on the specimen surface with the energy of $70 \mathrm{~mJ}$ and the thickness of water layer from the top surface of sample was $30 \mathrm{~mm}$. In addition, experiment was also carried out to investigate a capability to detect laser-induced breakdown by adjusting the laser to be focused in the water layer. Laser induced breakdown is a result from a reac-

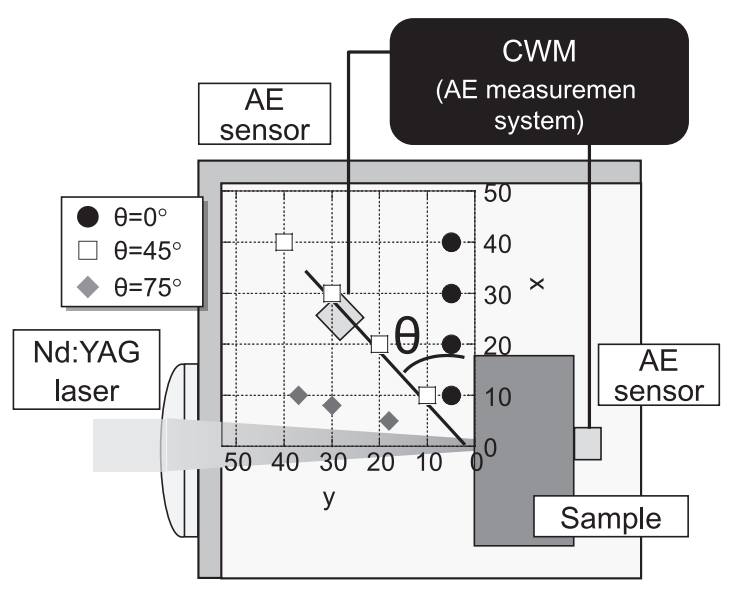

Dimensions: $\mathrm{mm}$

Fig. 2 Positions of the AE sensors in water in experimental setup 2.

tion of laser with confinement layer of water when the laser energy is high ${ }^{11)}$. The laser energy and water thickness were set to $100 \mathrm{~mJ}$ and $10 \mathrm{~mm}$ in this experiment, respectively. The second experimental setup is shown in Fig. 2. The surface of $35 \times 35 \mathrm{~mm}$ of the sample was set in the vertical direction and the laser was focused in the horizontal direction via a lens attached on the outside wall of the tank. With this arrangement the position of the AE sensor could be easily adjusted to evaluate the effect of sensor position on detected AE waveforms. The same AE sensor was used for all different positions to assure the same sensor property. The sensor was set at various angles $\left(0^{\circ}, 45^{\circ}\right.$ and $\left.75^{\circ}\right)$ and distances from the focused position. Another AE sensor was attached on the opposite side of the sample. These measurement results by both sensors were compared. The power density of laser was also varied for each sensor position while the condition of the water layer was kept constant.

\subsection{High speed camera observation}

A high speed camera (Phantom Miro M110, Vision Research ® Inc.), which has photograph speed of 220,000 fps and resolutions of $64 \times 64$ pixels, was used to observe sample surface during LSP. The times of high speed camera and AE measurement were synchronized. The phenomena observed by high speed camera were related to detected $\mathrm{AE}$ waveforms.

\subsection{Inverse analysis}

Impact force during LSP was evaluated by an inverse analysis of detected AE waveform. Detected AE waveform can be represented following equation,

$$
V(t)=S(t) * G(t) * I(t)
$$

where $*$ is represented convolution technique, and $V(t), S(t)$, $G(t), I(t)$ are detected waveform, response function of sensor, Green's function of sample and source function of impact force, respectively. $S(t)$ and $G(t)$ could be obtained from a simulation of AE waveform by finite element method and result of sharp pencil lead breaking experiment ${ }^{12)}$ and then $I(t)$ could be estimated by the deconvolution technique ${ }^{13)}$. Detected AE waveforms were analyzed by this inverse method and obtained impact force values were discussed. 


\section{Results and Discussion}

\subsection{Relation of detected AE waveforms and LSP phe- nomenon observed by high speed camera}

Figure 3 shows examples of AE waveforms detected during LSP from the sensor attached on the sample surface and the sensor fixed in the water layer. Figure 4 shows the LSP phenomenon on sample surface from the high speed camera observation. It should be noted that there was a time lag between the waveforms detected by both sensor settings because of the difference in the source-to-sensor distance of $20.0 \mathrm{~mm}$ for sensor on the sample and $60.0 \mathrm{~mm}$ for sensors in the water and wave velocity of 5100 and $1480 \mathrm{~m} / \mathrm{s}$ for Al alloy and water, respectively. This time lag was corrected and all waveforms were plotted at the same starting point to be easily related to the results observed by high speed camera.

By each laser irradiation, two AEs could be detected with a time lag of several hundreds microsecond. According to the high speed camera observation, after laser ablation (Fig. 4(a)),

(a)

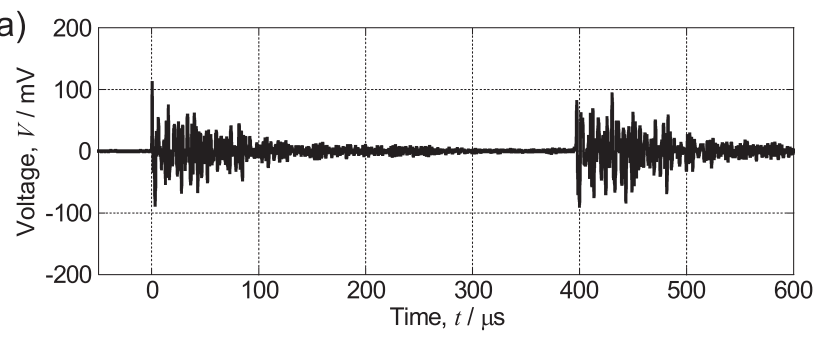

(b)

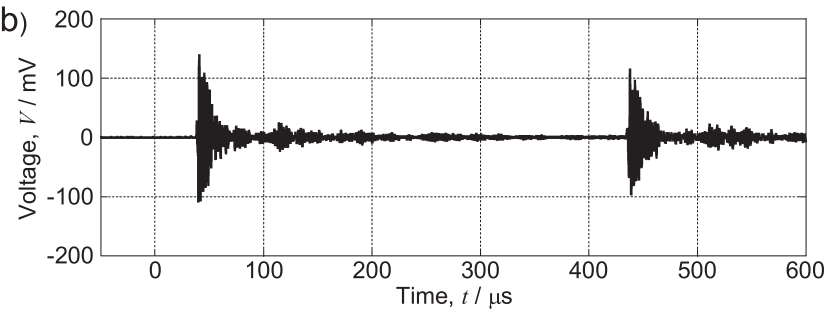

Fig. 3 Examples of detected AE waveforms during laser shock peening by (a) the sensor on sample and (b) the sensor in water.
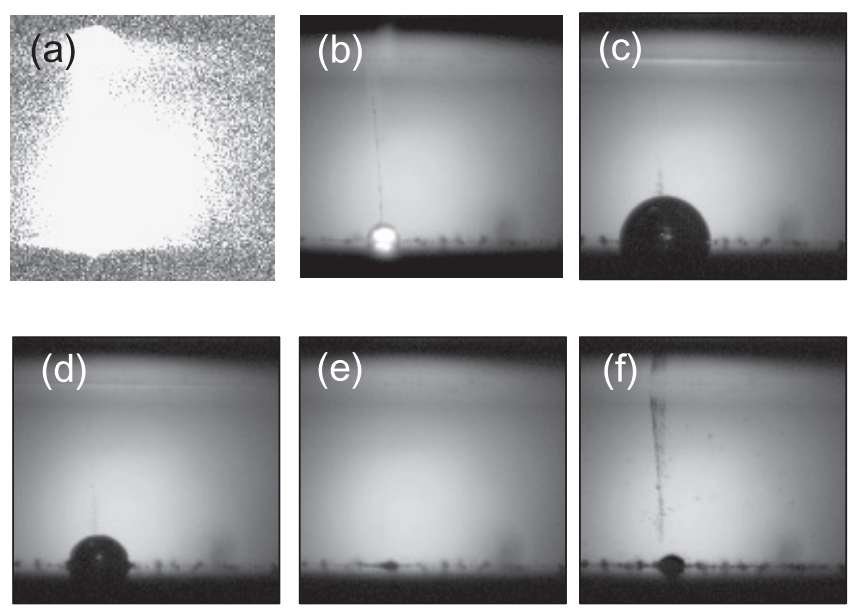

$5 \mathrm{~mm}$

Fig. 4 Observation of sample surface during laser shock peening by high speed camera at (a) $0 \mu \mathrm{s}$, (b) $13 \mu \mathrm{s}$, (c) $207 \mu \mathrm{s}$, (d) $350 \mu \mathrm{s}$, (e) $402 \mu \mathrm{s}$ and (f) $415 \mu$ s from laser irradiation. a bubble generated (Fig. 4(b)), expanded its size up to the maximum radius (Fig. 4(c)) and then became smaller (Fig. 4(d)) and finally collapsed (Fig. 4(e)). After bubble collapse, small bubbles in water layer were generated by shock wave passage (Fig. 4(f) $)^{14)}$. Since times of both high speed camera and $\mathrm{AE}$ measurement were synchronized, phenomena observed by high speed camera could be correctly related to detected AE waveforms. It was concluded that the sources of the former and latter AEs were laser ablation and bubble collapse, respectively. The waveform detected from both sensor settings showed the same results. It shows that monitoring of $\mathrm{AE}$ propagated in water is available to detect laser ablation and bubble collapse as same as monitoring of AE in sample.

\subsection{Detection of laser-induced breakdown}

The laser-induced breakdown was intentionally generated by focusing laser beam into the water, 5-mm higher from the sample surface with maximum laser energy in this experiment. Figure 5 shows examples of AE waveforms detected from both sensors located in the water and that attached on the specimen during laser-induced breakdown. At the same time, breakdown was also observed by the high speed camera as shown in Fig. 6. The photograph of 0- $\mu$ s (Fig. 6(a)) showed laser ablation occurred in the water. The noise signal observed in this photograph was due to laser light. After laser ablation, the bubble formed at the sample surface (Fig. 6(b)) and then became bigger (Fig. 6(c)) and finally collapsed (Fig. 6(e)) as described in the previous section. However, compared to the phenomenon shown in Fig. 4, where the laser was focused onto the sample surface, not only the bubble on sample surface was observed but there was also another bubble generated in the water as shown in the photograph of $65 \mu$ s (Fig. 6(b)). This bubble was generated by breakdown ${ }^{11)}$ and collapsed at $142 \mu$ s (Fig. 6(c)) while that generated on the sample surface collapsed at the $337 \mu$ s (Fig. 6(e)).

Consider the results from AE measurement, while the sensor attached on the sample surface could only detect two AE

(a)

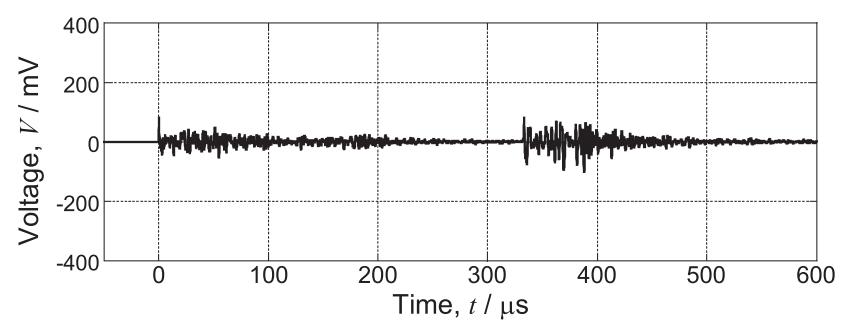

(b)

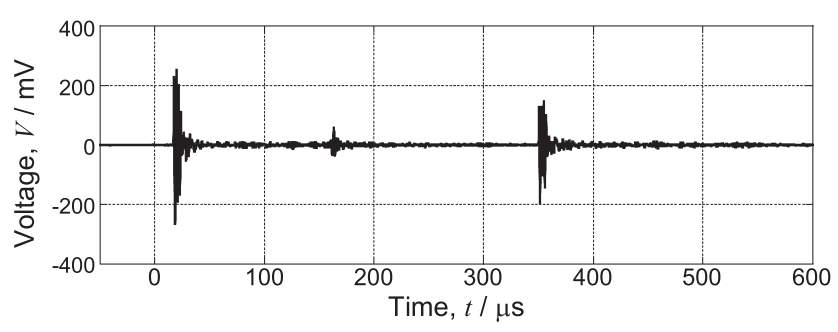

Fig. 5 Another examples of detected $\mathrm{AE}$ waveforms during laser shock peening (a) by the sensor on sample and (b) by the sensor in water. 

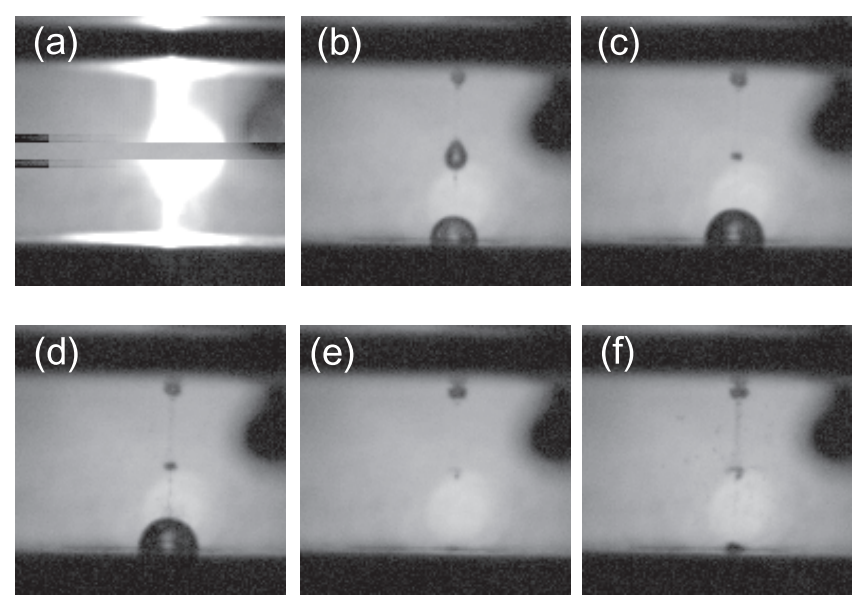

$5 \mathrm{~mm}$

Fig. 6 Observation of laser-induced breakdown during LSP by high speed camera at (a) $0 \mu \mathrm{s}$, (b) $65 \mu \mathrm{s}$, (c) $142 \mu \mathrm{s}$, (d) $155 \mu \mathrm{s}$, (e) $337 \mu \mathrm{s}$ and (f) $350 \mu$ s from laser irradiation.

waveforms generated from laser ablation and bubble collapse (Fig. 5(a)), those located in the water layer could detect another waveform at $142 \mu$ s (Fig. 5(b)). Since the time of high speed camera and AE measurement was synchronized, this $\mathrm{AE}$ waveform could be attributed to the collapse of bubble, which generated in the water. According to this finding, it is clearly shown that the generation of laser-induced breakdown can be detected between two prominent AE events. It should be noted that only sensor located in water layer could detect the AE from laser-induced breakdown. A possible explanation was that the bubble generated from laser induced breakdown had a small size and then an AE with small energy should be generated when it collapsed, for example the peak amplitude of this AE event was about $50 \mathrm{mV}$ from Fig. 5(b). This AE was difficult to be detected by the sensor attached on the sample surface because AE had to propagate from water into the sample. Most of signal might reflect at the sample surface.

It is clearly shown that monitoring by AE sensors set in the water is able to detect also the uncertain phenomenon during process such as laser-induced breakdown. Since laser-induced breakdown decreases laser energy for LSP, this detection is useful for a process control.

\subsection{Source location}

One benefit of using multi-channel AE measurement is an ability of AE source location. Sedlak et al. showed that AE source could be calculated from detected AE waveforms by attachment of several AE sensors on appropriate sample surface positions ${ }^{15}$ ). Based on the experimental setting in Fig. 1, $\mathrm{AE}$ source location was calculated from the difference in arrival time of AE events among four sensors and the wave velocity in the water. Observation of sample surface after laser irradiation at five different positions is shown in Fig. 7(a). Craters generated from laser ablation are clearly observed as black spots with diameters of $\sim 0.7 \mathrm{~mm}$. The results of source location on X-Y plane calculated from AE waveforms for both AEs generated from laser ablation and bubble collapse are shown in Fig. 7(b). A good agreement between the loca-
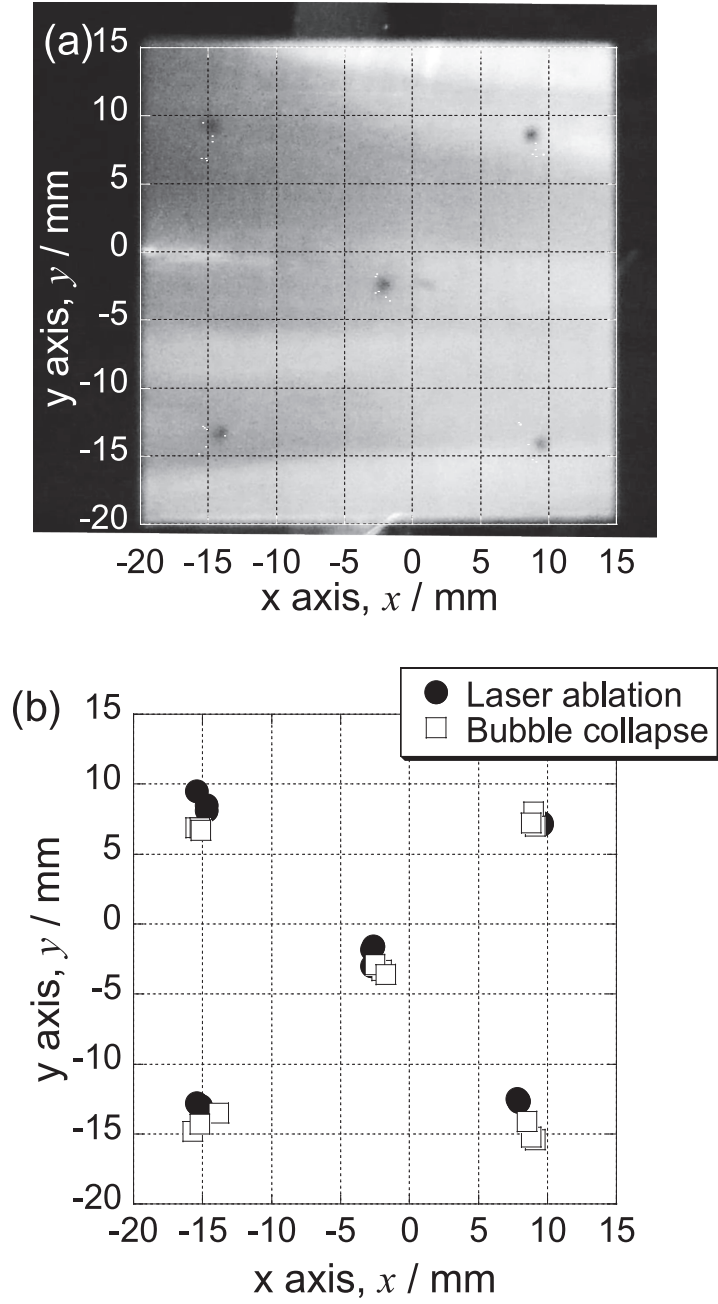

Fig. 7 Comparison between (a) photograph of peened sample and (b) source location by detected AE events.

tion results obtained from $\mathrm{AE}$ wave analysis and those observed the sample after experiment is confirmed. An average of location error in X-Y plane was less than $3 \mathrm{~mm}$. However, an average of location error in $\mathrm{Z}$ direction was approximately $5 \mathrm{~mm}$. Such a large error was attributed to the present sensor arrangement, where two of four sensors were located at the same $\mathrm{Z}$ position as the sample surface (Fig. 1(a)). This error can be minimized by optimization the sensor position. The present findings implied that using multi-channel AE measurement in the water layer provides good performance for in-process monitoring of LSP. The measurement by the sensor attached on the sample surface has several disadvantages such as influence of sample on the detected waveform, installing and detaching the sensor. The measurement by sensors set in the water overcomes these problems. The setting and changing the sample become easy because no need to attach and detach the AE sensor. There is little effect of sample on the detected waveform because the wave directly propagates to the sensor.

\subsection{Effect of sensor position}

The position of AE sensor affects the characteristic of detected waveform such as amplitude, etc. Plots of peak amplitude, $V_{p}$, as a function of source-to-sensor distance, $d$, for 
(a)

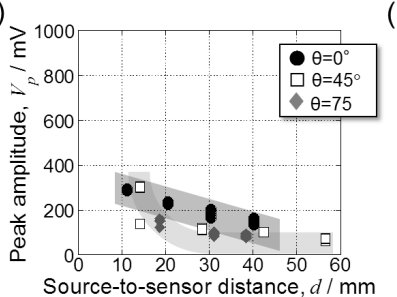

(b)

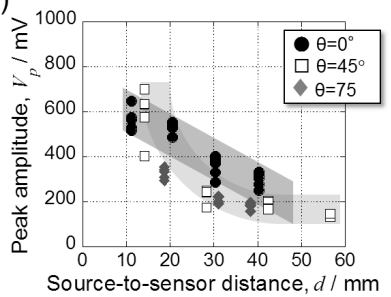

(c)

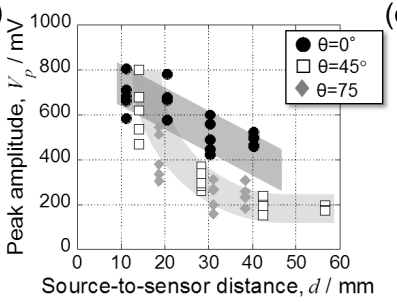

(d) $>$

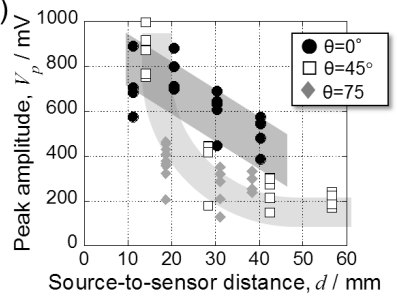

Fig. 8 Peak amplitude of detected AE by laser ablation at power density of (a) $0.45 \times 10^{4} \mathrm{GW} / \mathrm{m}^{2}$, (b) $1.5 \times 10^{4} \mathrm{GW} / \mathrm{m}^{2}$, (c) $3.0 \times 10^{4} \mathrm{GW} / \mathrm{m}^{2}$ and (d) $4.7 \times 10^{4} \mathrm{GW} / \mathrm{m}^{2}$

different power densities are given in Fig. 8. Here the peak amplitude was defined as the voltage of the first peak of detected waveforms. The peak amplitude tended to increase with power density and decrease with increasing source-tosensor distance for all angle positions. However, manners of amplitude decreasing were quite different depending on the position of the sensor. For example, a linear relation was observed at $\theta=0^{\circ}$ while exponential relation was observed at other angles. Figure 9 shows plots of the peak amplitude as a function of laser power density at source-to-sensor distances of 30 and $40 \mathrm{~mm}$. The peak amplitude increased with increasing laser power density and was influenced by the sensor position. The peak amplitudes in the case of $\theta=0^{\circ}$ showed higher values than those detected at $\theta=45^{\circ}$ and $75^{\circ}$ while the two latter ones showed the same values although there was a scattering. Since the source-to-sensor distances for all sensor positions were the same, the peak amplitudes should be the same values. The higher amplitude of the waveform detected at $\theta=0^{\circ}$ might be related to the interference of wave propagated in water and that propagated through the sample. The same values for peak amplitudes detected at $\theta=45^{\circ}$ and $75^{\circ}$ inferred that only waves propagated in water were detected. These findings implied that sensor position influences the detected waveform and using only peak amplitudes of detected $\mathrm{AE}$ events is not appropriate to evaluate LSP behavior.

\subsection{Pressure estimation from inverse analysis}

As shown in the previous section, only the peak amplitude cannot be used to evaluate the LSP process because the detected waveforms were influenced by the sensor position. The inverse analysis is a solution for this problem. According to eq. (1), $S(t)^{*} G(t)$, which was determined from pencil-lead breaking test and FEM analysis for each sensor position. An example of impact force after inverse analysis from detected wave of sensor at $(x, y)=(10,10)$ was showed in Fig. 10. Pressure was calculated from the peak impact force as Fig. 10 and the area irradiated laser. The pressure values obtained from the inverse analysis from the sensor set on the sample surface and that set in the water at $\theta=45^{\circ}$ are shown in Fig. 11. It is noted that $(x, y)$ in this figure shows positions of

(a)

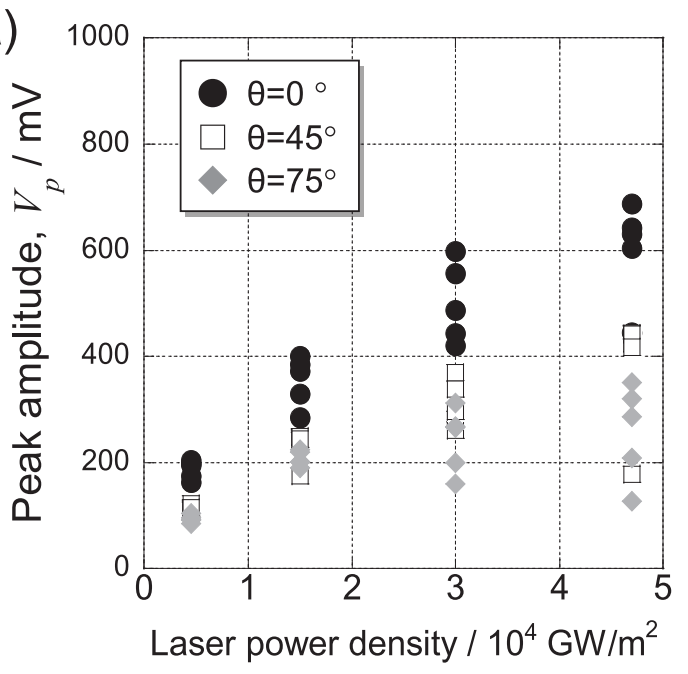

(b)

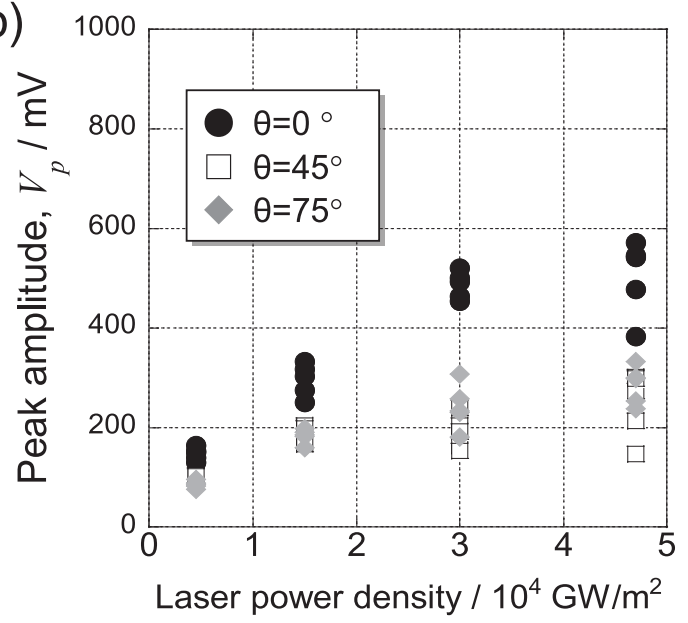

Fig. 9 Peak amplitudes of detected AE s by laser ablation at distances of (a) about $30 \mathrm{~mm}$ and (b) about $40 \mathrm{~mm}$.

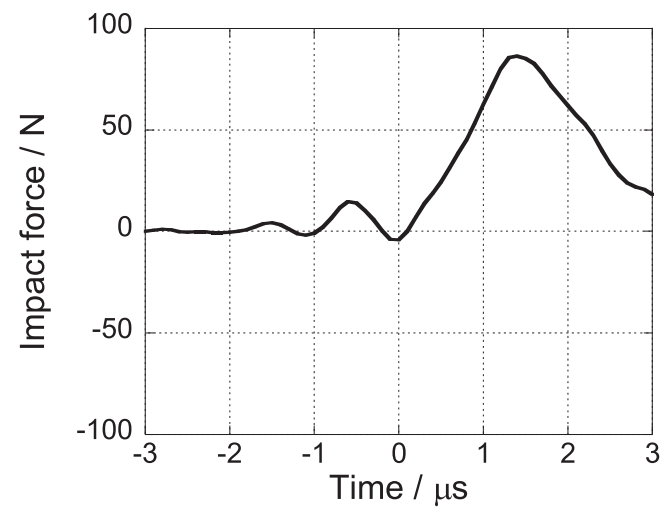

Fig. 10 An example of Impact force after inverse analysis.

AE sensor. $P_{w}$ represents pressure calculated from detected AE waveform by the sensor in water and $P_{s}$ represents pressure from detected waveform by the sensor on sample. Since there were two waveforms generated from laser ablation and bubble collapse, the pressure of the former and the latter ones are shown in Fig. 11(a) and (b), respectively. The pressures from laser ablation calculated from both sensors were in good agreement (Fig. 11(a)). It seemed that the scattering of pres- 

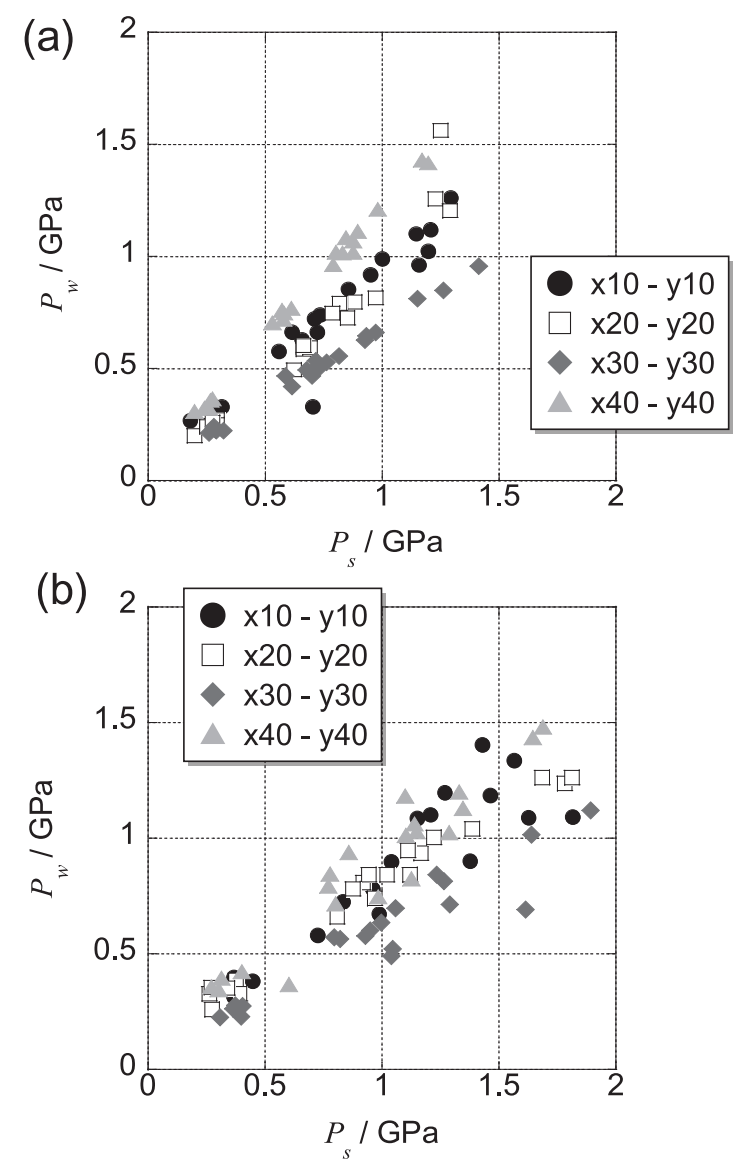

Fig. 11 Comparison of pressure analyzed from AEs propagated in sample with those propagated in water of (a) laser ablation and (b) bubble collapse.

sure became pronounced with increasing power density. An increase of absolute value of the pressure leaded to an increase of the error even though the error ratio was same. The improvement of experimental setting is required to minimize these errors. The pressures from bubble collapse calculated from both sensors were also in good agreement (Fig. 11(b)), however, the pressures obtained from the sensor set in the water layer seemed to give lower values than those obtained from the sensor attached on the sample when increasing power density. There were different between positions of $\mathrm{AE}$ sources because laser ablation was generated at sample surface while a cavitation bubble was collapsed in the water layer near sample surface. It was thought that this difference of position affected on AE propagation in both sample and water layer. Therefore, there were also different tendency in the relationships between pressures in the comparisons of analysis results of laser ablation and bubble collapse. Although the results obtained from $\theta=0^{\circ}$ and $75^{\circ}$ were not shown here, the magnitude of pressure values and tendency were similar to those observed when $\theta=45^{\circ}$.

It should be noted that the plot of peak amplitude as shown in Fig. 8 and 9 showed a strong effect of sensor position. However, there were small effects on pressure in Fig. 11. Data in this figure were liner fitted for each sensor position and slopes and correlation coefficients of fitted liner relationship were derived. This analysis was also performed on pressure data obtained from $\theta=0^{\circ}$ and $75^{\circ}$. Plots of slope $\left(P_{w} / P_{s}\right)$
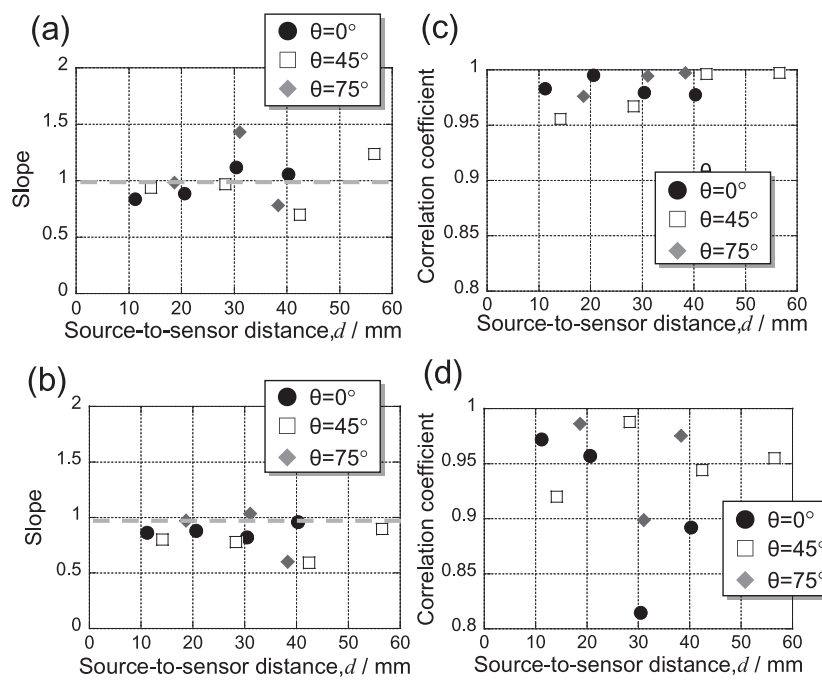

(d)

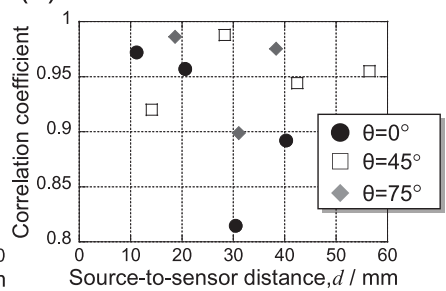

Fig. 12 Slope of the relationships between $P_{s}$ and $P_{w}$ by (a) laser ablation and (b) bubble collapse, and correlation coefficient of the relationships between $P_{s}$ and $P_{w}$ of (c) laser ablation and (d) bubble collapse as Fig. 10.

and correlation coefficients as a function of source-to-sensor distance are shown in Fig. 12. As shown in Fig. 12(a) and (b), slope of pressure of laser ablation and bubble collapse such as Fig. 11(a) and (b) was near unity. The result shows monitoring of AE propagated in water is possibility to evaluate LSP in the same way as that of AE propagated in sample. On the other hand, correction coefficient of bubble collapse was smaller than that of laser ablation (Fig. 12(c) and (d)). The reason is because the differences among impact sources by laser ablation, bubble collapse and pencil lead breaking of calibration affected on propagation of shock waves.

Although there were scattering in the data, the pressure tended to be liner relation with power density for all sensor positions. Furthermore, the almost pressure values could be obtained independent on the sensor position. Therefore, it can be implied that the inverse analysis was able to correct the differences between detected AE events at each sensor position in the water layer. It is thought that gaps between value of pressure calculated from detected AE events by the sensor attached sample and the sensor in water could be corrected in future study.

\subsection{Performance of AE monitoring in water for LSP}

According to several findings in the previous sections, it is shown that setting AE sensors in the water instead of direct attaching on the sample surface provides various benefits such as capability of location, laser-induced breakdown detection, and easy installation. The pressure values estimated from the inverse analysis from the AE events detected from various sensor positions also showed a good agreement with those obtained from the sensor attached on the sample surface. This infers that monitoring of AE propagated in water is a powerful tool for LSP. The on-line process monitoring to control the quality of LSP is expected to be available by this technique. 


\section{Conclusions}

The LSP was quantitatively evaluated by monitoring of AEs propagated in both the water and the sample coupling with high speed camera. The findings obtained from the present research are as follows:

(1) Instead of direct attachment of the AE sensor on the sample surface, fixing multiple sensors at various positions in the water layer was investigated for AE monitoring. The results showed that $\mathrm{AE}$ waveforms could be detected by this new arrangement. A good source location was obtained with a small error.

(2) Generation of laser-induced breakdown could be clearly detected by setting AE sensors in water.

(3) Although detected AE waveforms showed different behaviors depending on the sensor position, the pressure values could be calculated from the inverse analysis of these detected waveforms.

\section{REFERENCES}

1) L.M. Barker and R.E. Hollenbach: J. Appl. Phys. 43 (1972) 4669-4675.

2) M. Gelfi, E. Bontempi, R. Roberti and L.E. Depero: Acta Mater. 52 (2004) 583-589.

3) M.G.R. Sause and S. Horn: J. Acoust. Emiss. 28 (2010) 142-154.

4) L. Lin and F. Chu: Mech. Syst. Signal Process. 26 (2012) 181-189.

5) Q. Wang and F. Chu: J. Sound Vib. 248 (2001) 91-103.

6) T. Kundu, S. Das, S.A. Martin and K.V. Jata: Ultrasonics 48 (2008) 193-201.

7) N. Choi, T. Kim and K.Y. Rhee: NDT Int. 38 (2005) 268-274.

8) D.E. Lee, I. Hwang, C.M.O. Valente, J.F.G. Oliveira and D.A. Dornfeld: Int. J. Mach. Tools Manuf. 46 (2006) 176-188.

9) M. Enoki, K. Kobayashi, T. Tomoki, M. Matsui and Y. Kobayashi: EWGAE 2012.

10) K. Ito and M. Enoki: Mater. Trans. 48 (2007) 1221-26.

11) B. Wu and Y.C. Shin: Appl. Phys. Lett. 88 (2006) 041116

12) M.G.R. SAUSE: J. Acoust. Emiss. 29 (2011) 184-196.

13) D.J. Buttle and C.B. Scruby: Wear 137 (1990) 63-90.

14) C.D. Ohl: Phys. Fluids, 14 (2002) 3512-3521.

15) P. Sedlak, Y. Hirose, S.A. Khan, M. Enoki and J. Sikula: Ultrasonic 49 (2009) 254-262. 